\title{
Electronic Supported Solutions to Scale-up Maternal New-born and Child Health Interventions Feasibility Assessment for Hoima District
}

\author{
Geoffrey Babughirana ${ }^{1,}$, , Joel Fred Nsumba ${ }^{1}$, Elisha Nangosha ${ }^{1}$, Benon Musasizi ${ }^{1}$, \\ Rebecca Mirembe Mulindwa ${ }^{1}$, Brian Manyasi ${ }^{2}$, Lucy Adeke $^{3}$ \\ ${ }^{1}$ World Vision International, Kampala, Uganda \\ ${ }^{2}$ Independent Scholar, Kampala, Uganda \\ ${ }^{3}$ Programu, Kampala, Uganda
}

Email address:

gbabughirana@gmail.com (G. Babughirana)

${ }^{*}$ Corresponding author

\section{To cite this article:}

Geoffrey Babughirana, Joel Fred Nsumba, Elisha Nangosha, Benon Musasizi, Rebecca Mirembe Mulindwa, Brian Manyasi, Lucy Adeke. Electronic Supported Solutions to Scale-up Maternal New-born and Child Health Interventions Feasibility Assessment for Hoima District. International Journal of Information and Communication Sciences. Vol. 6, No. 1, 2021, pp. 11-21. doi: 10.11648/j.ijics.20210601.12

Received: February 26, 2021; Accepted: March 13, 2021; Published: April 7, 2021

\begin{abstract}
Background: The application of mobile telecommunication technologies in the improvement of Maternal New-born and Child Health for developing countries including Uganda is one of the key innovations in improving health outcomes. One of the key models used in disseminating health messages to mothers is the timed and targeted counselling (ttC) model. This model is being implemented in Uganda and it utilizes the services of Village Health Teams (VHTs) to drive change through mHealth. Understanding the feasibility of electronic supported solutions in scaling up Maternal New-born and Child Health intervention is critical at inception to guide resource allocation, understand existing strength, weaknesses, opportunities and threats to successful implementation of a mobiles health solution. Methods: Across-sectional study to collect quantitative and qualitative data from 432 VHTs was used. A census of 43 Health facilities was also done in Hoima district. Secondary data was collected from the District Health Team, Ministry of Health $(\mathrm{MoH})$ and other relevant actors. The study followed the mHealth Assessment and Planning for Scale (MAPS) Toolkit self-assessment and planning guide designed to offer improvement of project capacity to pursue strategies that increase potential for scaling up and long-term sustainability achievements. A semi structured questionnaire was used which assessed the mHealth feasibility basing on the 16 domains under the 6 axes for the assessment for scale-up of an mHealth platform. Data was analysed at different levels from CommCare, and the District Health Information System (DHIS2) using SPSS. Results. 95.8\% of VHTs owned a mobile phone and majorly used them for text messaging (71.3\%). The biggest portion of VHTS use MTN network for voice calls or data services with accessibility findings of $88.8 \%$ and $86.2 \%$ respectively. Most health facilities $(55.8 \%)$ had personnel trained in computer skills but only $46.5 \%$ are proficient in basic computer skills. From the analysis of the 6 axes, the functionality of the mHealth in Hoima district was at $42.6 \%$ with Axis 1 on ground work planning and implementation scoring highest with $63.9 \%$ and Axis 3 that focuses on Financing the mHealth platform scoring the lowest of $30.0 \%$. Conclusion. For successful application of telecommunication mobile technologies, partnership between different actors needs to be up scaled to reduce on costs and maximize benefits. A deeper stakeholder analysis needs to be done for different organisations to be engaged in different elements that support the mHealth project.
\end{abstract}

Keywords: mHealth, e-health, Scale-up Assessment, Domains, Axis, VHT Readiness, Health Facility Readiness, Functional Analysis

\section{Introduction}

The timed and targeted counselling (ttC) model that involves key health and nutrition messages disseminated to pregnant, and breastfeeding mothers and their families to cause behavioral change at specific timelines in the 1,000 days [1]. The ttC-Village Health Teams (VHTs) make a series of 
visits to the households organizing all the 7-11 messages [2] into "timed and targeted" sets communicated at the most appropriate times, using a counselling and dialogue-based approach. The ttC approach has been transformed by World Vision from the paper based to an electronic system which has gone mobile through the Mobile Health (mHealth) CommCare innovation [3]. The CommCare (MOTECH [4] suite solution) is an open source mobile tool based on the ttC strategy, and a component of the software most visible to the end user operating the mobile set. This is utilised as a mobile App which relays data collected from VHT household sessions in real time to the CommCare Dashboard for real time analysis and decision making for program support.

Mobile telecommunication technology is increasingly becoming an important tool in global health programs. There is excitement about the potential of "mHealth centres" focusing on how mobile technology is utilised in lower and middle-income countries where people have traditionally had limited access to health services [5]. mHealth strategies are being used to overcome factors that limit access, such as geographic distance to services, social marginalization, inadequate skilled medical personnel or a lack of financial resources [6]. The CommCare is uploaded in the mobile phones as a community system strengthening approach to improve Maternal, New-born and Child Health (MNCH) [3]. The mHealth project outfitted the VHTs with mobile phones enabled with the CommCare application to address the $\mathrm{MNCH}$ issues among the most in need and vulnerable

\section{beneficiaries in Hoima district.}

To comprehensively build case and make sense for the scaling-up of the mHealth project in Hoima district, an assessment exercise based on the World Health Organization (WHO) recommended practices for scaling up mHealth was adopted. These are believed to be the core influencers for the scaling-up of mHealth in any given locality [7]. This assessment allows for local, national and global level stakeholders and players to assess their stand in relation to each of the recommended practices and to identify areas that require attention and devises strategies to overcome challenges or obstacles to the desired scaling-up [8].

In this regard, the study followed the mHealth Assessment and Planning for Scale (MAPS) Toolkit a comprehensive self-assessment and planning guide designed to improve the capacity of projects to pursue strategies that increase their potential for scaling up and achieving long-term sustainability [9]. MAPS are designed for project teams who are already deploying mHealth, aiming to increase the scale of impact. Stakeholders seeking to understand the value of mHealth also find value in using the Toolkit jointly with project teams.

The Toolkit covers six major areas (referred to as the "axes of scale") that determine the scaling up of mHealth: Groundwork, Partnerships, Financial health, Technology \& architecture, Operations, and Monitoring \& evaluation. The axes of scale represent the key concerns, activities and decisions that relate to these six areas [9]. The axels are scored by points from domains as seen in the table 1 Below:

Table 1. MAPS Axels and their domains of scale.

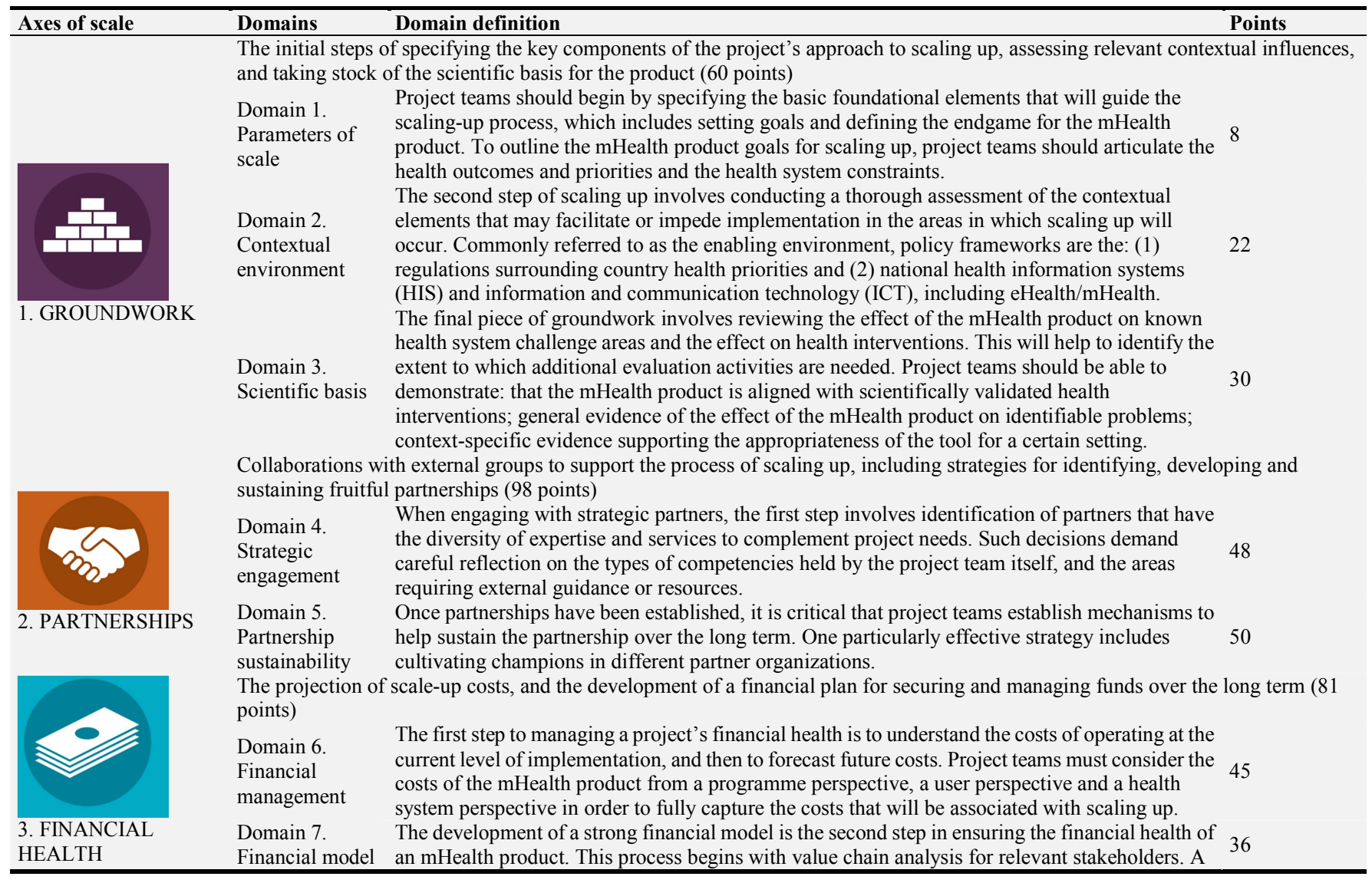




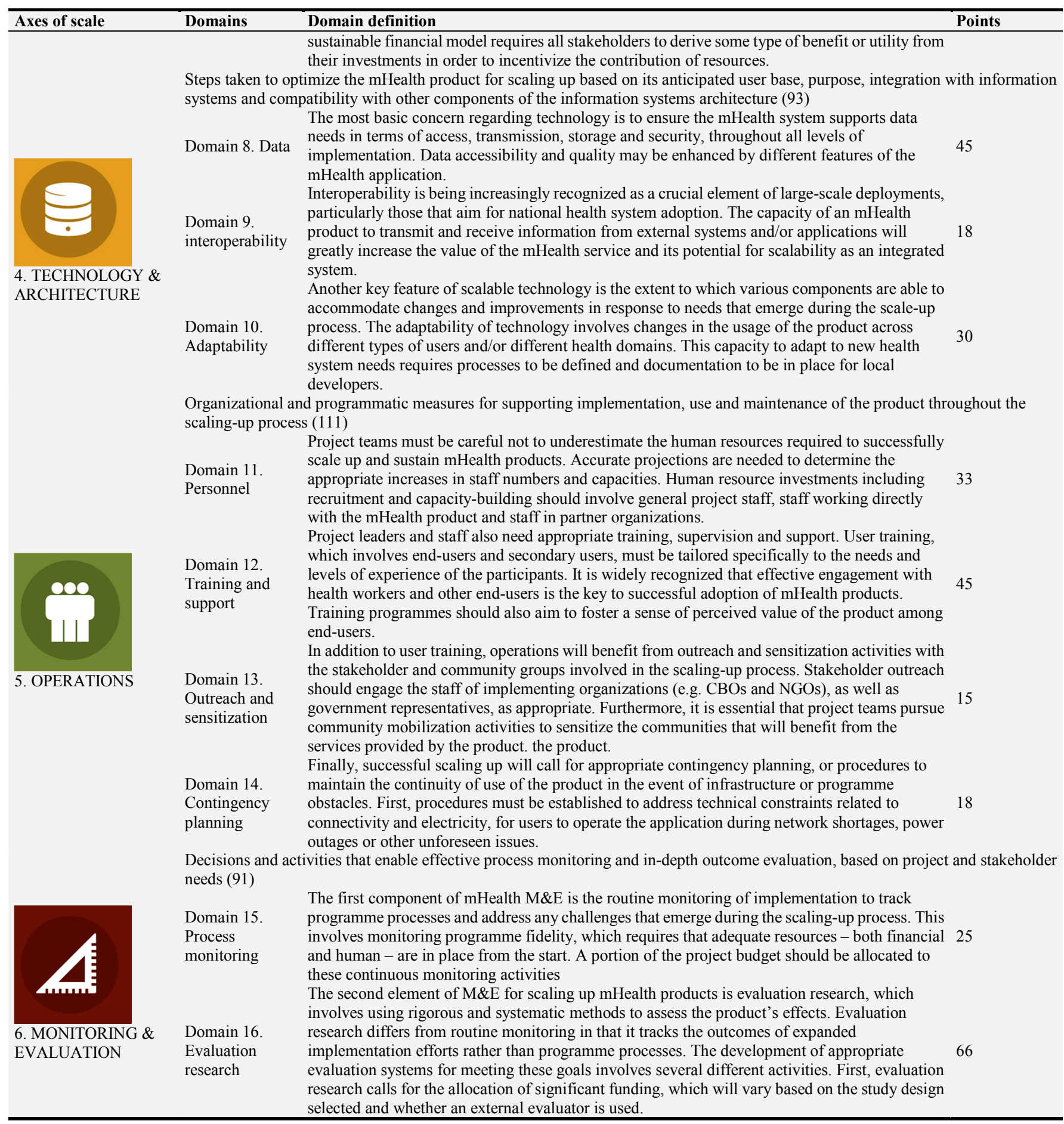

MAPS allow assessment to ascertain where projects stand in relation to each of the axes of scale, and tracks progress as activities evolve. The Toolkit helps project teams to identify areas that require further attention, and to devise strategies to overcome any challenges to progress. MAPS are designed to be used periodically at several time points throughout a project's trajectory, guiding projects through an iterative process of thorough assessment, careful planning and targeted improvements. These steps facilitate successful scaling up of mHealth products.

By the close of 2016, Uganda was still struggling with key
Maternal New-born and Child Health indicators that contribute to maternal and child Survival. For Example, 40\% of Ugandan pregnant women do not make the required 4 visits before delivery, with $27 \%$ of women still delivering at home [10]. Further still a study carried out in 3 districts of Western Uganda revealed a neonatal mortality of 34 per 1000 live births with actors associated with increased neonatal deaths included presence of new-born danger signs while factors associated with lower risk of neonatal death included home visits by community health workers and attendance of at least 4 antenatal visits [11]. 
With the above revelations and challenges, studies elsewhere have pointed to the positive contribution of mobile phone intervention in most of the selected $\mathrm{MCH}$ service indicators, like improvement in the percentage of recommended ANC visits, percentage of deliveries attended to by trained health workers and also facilitate the work processes of the health workers in rural areas [12]. It's the basis of this evidence that this project took a strategic direction to implement mHealth and hence the need for a baseline assessment to facilitate evaluation of feasibility of the mHealth project with focus on providing vital information on network mapping, district preparedness and functionality of the system. This was anticipated to inform project implementation through informing, tracking, and documenting of performance. The Purpose of the study is to assess the current mHealth initiatives with in Hoima district linked to the maternal newborn and child health programming. Superficially focusing on the gaps, and transform these into key actions to scale districtwide.

Conceptual Framework

The baseline survey process is pivotal in the conceptual framework of the Maternal newborn and child health project. Figure 1 below shows how mHealth and innovations around information technology for development (ICT4D) fits into the project cycle for the achievement of key target outcomes in $\mathrm{MNCH}$. If the $\mathrm{MNCH}$ project in Hioma implements the principle of a VHT system that is available and functional, and sets appropriate targets depending on the key struggling $\mathrm{MNCH}$ indicators, then the Project should anticipate a positive trend with these indicators. as long as the mHealth assessment focuses on the status of the network coverage, information volumes, interoperability and integration with government existing platforms. The project should further focus on readiness to invest in components that require human and financial resources. The mHealth assessment aims to ensure that the intermediary catalytic approaches for mHealth are ascertained focusing on how they will be handled and achieved in the long run. This will be in order to ensure that the key $\mathrm{MNCH}$ outcomes are achieved. This is further illustrated below in figure 1 below:
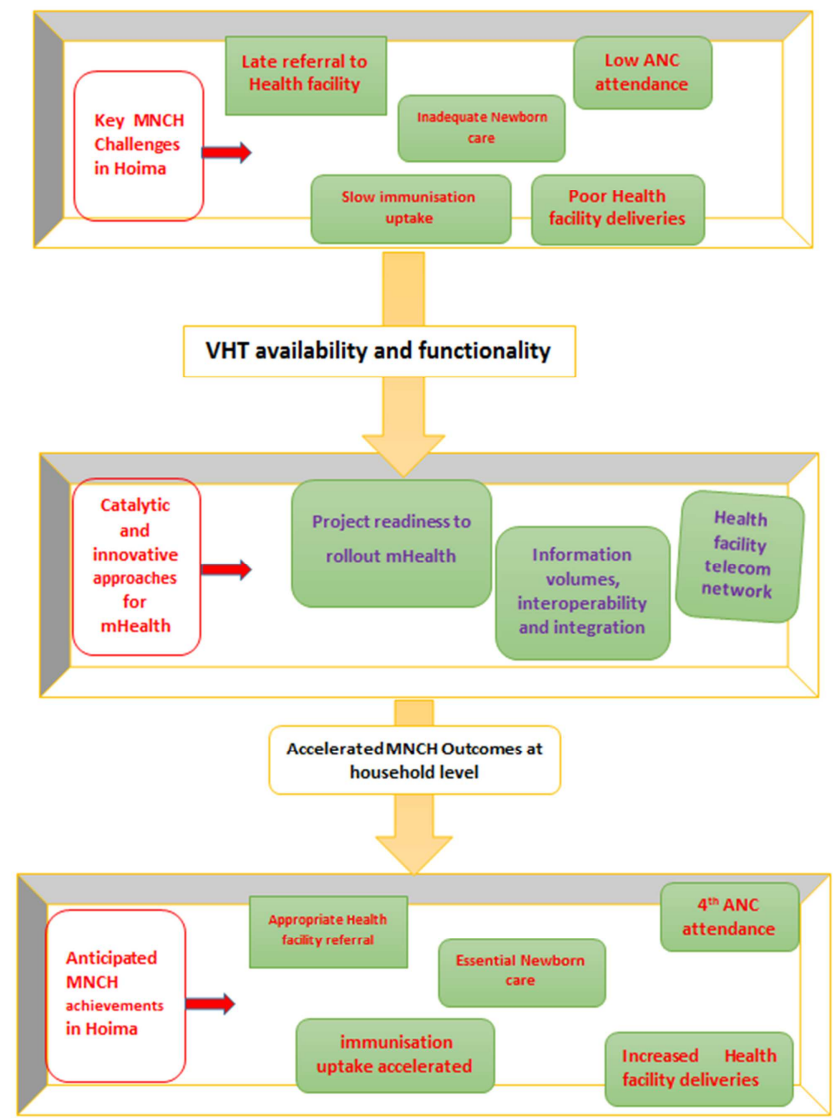

Figure 1. Conceptual framework for the implementation of mHealth in the MNCH project Hoima.

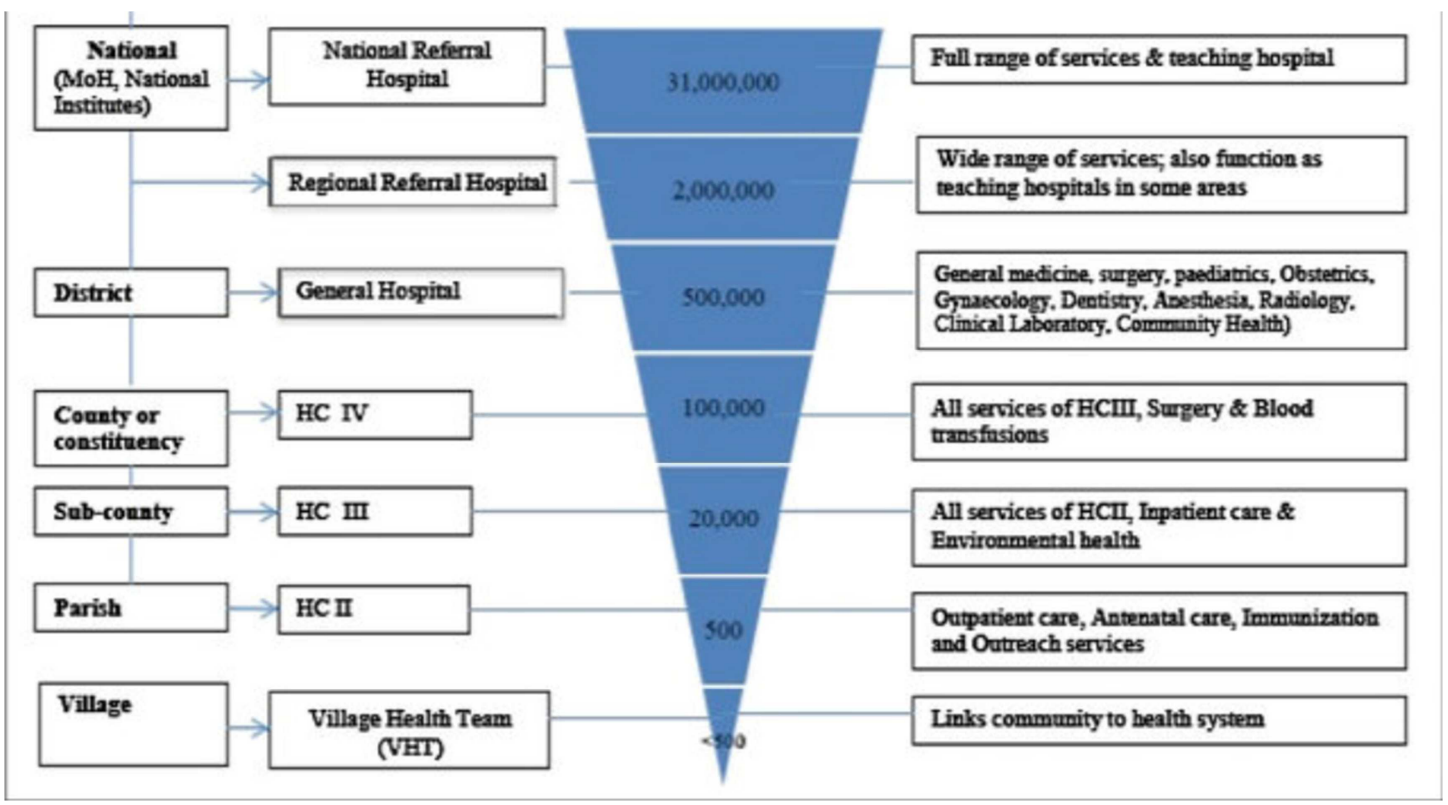

Figure 2. Health facility level and population served [16]. 


\section{Methodology}

\subsection{Study Area}

Situated in the Western Region of Uganda, Hoima District is bordered by Buliisa District to the north, Masindi District to the northeast, Kyankwanzi District in the east, Kibaale District to the south, Ntoroko District to the southwest and the Democratic Republic of the Congo across Lake Albert to the west. The coordinates of the district are: $0124 \mathrm{~N}, 31$ 18E [13]. Administratively it is divided into 2 Counties, 1 Municipal Council, 4 Divisions, 1 Town council, 15 sub counties and 558 villages. The health facility landscape is furnished with 57 government health facilities (1 hospital, $4 \mathrm{HC}$ IVs, $32 \mathrm{HC}$ IIIs and $20 \mathrm{HC}$ IIs), 9 private-not-for-profit health facilities and 4 private-for-profit health facilities [14].

The Health facilities in Hoima are based on the Health facility structure of Uganda and guided by the Health sector strategic and investment plan [15]. Uganda's healthcare system operates on an upward referral basis; for example, when a level II health center cannot handle a case, it refers it to the next level as detailed below:

\subsection{Survey Design}

The baseline survey and feasibility assessment used a multifaceted design consisting of quantitative and qualitative methods. Both primary and secondary data was collected from identified respondents from the sampled VHTs and DHTs; World Vision Uganda (WVU) national and Hoima office, $\mathrm{MoH}$ and other relevant stakeholders. This was done using two questionnaires designed for the baseline survey; one for the personnel at the HFs and another for the VHTs. Personal interviews guided by the questionnaire were held with the VHTs and HF staff.

The questionnaire for the assessment of mHealth feasibility was a standardized assessment tool and it was shared with the personnel of interest prior to the actual scheduled date. This self-assessment questionnaire was not only shared as a hard copy document but also as a link within which responses to the questions could be made (online survey). The questionnaire consisted of questions relevant to the 16 domains under the 6 axes for the assessment for scale-up of an mHealth platform [17].

\subsection{Sample Size}

The Kish-Leslie formula [18] was used to determine the required VHT sample size.

$$
\mathrm{n}=\frac{\mathrm{D} \times \mathrm{Z}_{\alpha / 2}^{2} \mathrm{PQ}}{\delta^{2}}
$$

Where $n=$ required sample size, $D=$ design effect, $Z_{\alpha 2}=1.96$ (Critical value of the standard normal distribution corresponding to error rate $\alpha / 2$ at the level of significance $\sigma=0.05(5 \%)$,

$\mathrm{D}=1$ (the design effect is 1 as widely used by other studies)

$\mathrm{P}=\mathrm{A} 50 \%$ estimated population proportion of correct use of the m-Health commCare technology among VHTs was utilized to give the most conservative sample size as an estimate was unavailable as this was the first time in Hoima to conduct a m-Health CommCare baseline study on.

$\mathrm{Q}=(1-\mathrm{P})$, which represents the estimated proportion of VHTs who have knowledge on mHealth CommCare approach and functionality.

$$
n=D * \frac{Z^{2} P Q}{\delta^{2}}=\frac{1 * 1.96^{2} *(0.5 * 0.5) x^{2}}{0.05^{2}}=380
$$

$\mathrm{n}=380$ Village Health Workers

Non-respondents $=5 \%$

$\mathrm{n}=380+(5 \%$ of 380$)=395$ respondents

There was a census for the health facilities. At the data collection time, a total of 475 persons were respondents; this comprised of 432 VHTs and 43 HF personnel. A minimum of 9 VHTs were selected as respondents per parish and one personnel at the HF was selected as a respondent.

\subsection{Ethics Approval and Consent to Participate}

Ethical approval and clearance received from the Institutional Review Board (IRB) for the School of Public Health, Makerere University College of Health Sciences, the Higher Degrees, Research and Ethics Committee (HDREC) protocol 217 on the $19^{\text {th }}$ February 2019. All participants provided written consent to participate in the study.

\subsection{Data Management and Analysis}

The CommCare, mHealth and the District Health Information System (DHIS2) systems were analysed as part of the MAPS tool kit assessment. These systems were studied so as to ascertain functionality, interoperability, adaptability, data collected by the systems and the supporting software environments of these systems. They were studied to ascertain scale up and use in the Hoima district.

In addition to system studies, the network and internet coverage provided by mobile network operators (MNOs) was analysed at Sub County and HF level based on data collected. The baseline survey data was checked for accuracy, completeness, relevance and consistency of the critical data elements before it was analysed. The cleaned data was then entered into the IBM SPSS Statistics 2019 v26 for subsequent analysis.

The assessment for scale-up data was collected using an online survey platform and analysed using this platform. There was no need to export the data for further analysis as the platform has an inbuilt data analysis module.

\subsection{Analysis of the mHealth Scale-Up Assessment Findings}

The mHealth assessment for scale-up was based on a standard model. The self-assessment questionnaire consisted of 16 domains grouped into 6 axes on which consolidated scores were derived. Basing on the responses made in the online survey self-assessment questionnaire, weights were awarded per domain and consequently, per axis. 
The MAPS tool kit was used to facilitate a self-assessment of the current operations of mHealth with in Hoima health sub-district [9]. The Focus was on the persons who take on the role of the mHealth project manager, other relevant members of the project team and the members in the District health office who deal with data. The assessment followed the two suggested approaches for completing and responding to the self-assessment questions (SAQ):

Individual assessment: Different members of the $\mathrm{HMNCH}$ team were targeted to complete different sections of their concern and experience. Certain sections required some responses from individuals with particular skill sets within the project; e.g. the financial manager for Axis 3 (Financial health) and the information technology team for Axis 4 (Technology $\&$ architecture). The data collection team them held hold a meeting with the entire team to review and agree on the results.

Team assessment: This was done to involve the Hoima district Health team focusing on those who are involved in HMIS, DHIS and any mHealth activities. This was a whole day meeting aimed at confirming or making changes in the scores based on the options of the current implementation for mHealth.
The Toolkit took approximately $1.5-2$ hours to complete in its entirety. Determining the next steps addressed any project shortfalls however highlighted by MAPS was a more extensive process.

The Toolkit was in this case used as a baseline assessment of where Hoima stands with mHealth and these were to be used to inform planning for improvement through the scaling-up process. Each SAQ has a series of sub-statements that capture the particular activities, considerations and procedures reflecting the relevant topic. Each sub-statement is accompanied by a set of response options. Some questions have the basic response options of No and Yes. However, the majority of the SAQs have four response options: No, In progress, Performed and Documented.

Participates used the guidelines shown in Table 2 to select the most appropriate response for each of the four options. The table includes examples showing how particular project experiences would align with the various response options.

In addition, a small number of questions had the option Not applicable (N/A), which means that the particular item does not apply to a project due to the context or the product. Finally, a few questions had a unique set of response options that are self-explanatory based on the content provided.

Table 2. Definition of scores.

\begin{tabular}{|c|c|c|}
\hline Option & Definition & Example of interpretation \\
\hline No & You have not addressed this item at all. & You have not considered the issues associated with electricity coverage at all. \\
\hline In progress & $\begin{array}{l}\text { You have begun to address this item, but further steps are } \\
\text { needed. }\end{array}$ & $\begin{array}{l}\text { You have gathered information about the access to and the consistency of } \\
\text { electricity in the settings where the product will be scaled up, but you have not } \\
\text { yet decided on how you will deal with these issues. }\end{array}$ \\
\hline Performed & $\begin{array}{l}\text { You have addressed this item fully, leaving no remaining } \\
\text { uncertainties. }\end{array}$ & $\begin{array}{l}\text { You have decided that you will provide health workers with solar chargers to } \\
\text { enable them to charge their mobile phones when in the field. }\end{array}$ \\
\hline Documented & $\begin{array}{l}\text { There is written documentation or evidence } \\
\text { demonstrating that this item has been completed. This } \\
\text { may include a report, or involve the development of } \\
\text { standard operating procedures (SOPs). }\end{array}$ & $\begin{array}{l}\text { You have documented the information needed for the provision of solar } \\
\text { chargers, including how many chargers will be necessary for each group of } \\
\text { health workers, the name of the vendor that will supply the chargers, and the } \\
\text { total cost. }\end{array}$ \\
\hline
\end{tabular}

After completing the MAPS Toolkit, the study team was able to calculate scores on three levels:

The Overall Score (Total Score Combining All Axes)

Axis scores (a separate score for each of the six axes of scale)

Domain scores (specific scores for the domains within each axis of scale).

The scoring mechanisms allowed the study teams to use quantitative terms to describe the overall progress through the process of scaling up, as well as the internal strengths and weaknesses. The opportunity to compare scores across axes and domains helped the $\mathrm{HMMCH}$ project teams determine which areas need further development.

Allocation of points: Points were allocated at the level of the sub-statements within each question. Each response option is worth a specific point value, as indicated in the check boxes. For example, $\mathrm{No}=0$ points, In progress $=1$ point, Performed $=2$ points, and Documented $=3$ points. Therefore, each question is worth a certain number of points depending on the number of sub-statements accompanying the question. The maximum number of points that can be allocated is indicated beside each question and each domain title.
Scorecards: Six scorecards - one for each axis of scale are provided to help users calculate their scores. The specific steps entailed in the scoring process are detailed in these scorecards.

Final scorecard: Ultimately, the calculations yielded a percentage for each axis of scale as well as for each domain. The final scorecard allowed users to compare scores across the axes of scale and the domains.

Its anticipated that the results obtained from the SAQs will be applied in several different ways. First, the axis and domain scores will help to shed light on the particular areas that may require additional consideration, activities and strategies from project managers and teams. The $\mathrm{HMNCH}$ project will consult the tips, lessons from the field, and resources provided in the planning and guidance section of each axis in order to plan efforts to address problem areas. In addition, since the Toolkit can be used periodically, the $\mathrm{HMNCH}$ project will employ it to assess and correct the course of their progress regarding scaling up and the pursuit of their endgame. 


\section{Study Results}

\subsection{Social Demographic}

A total of 432 VHTs participated in the survey across Hoima district. The district consists of 15 sub counties. Majority $(47 \%)$ of the respondents were from Kitoba Sub County which had the highest number of parishes. Whereas the majority of the respondents were female, the age categories of 31 through 40 and 41 through 50 represented the highest number of respondents. Out of the $56 \%$ female respondents, the majority $(30.58 \%)$ were in the age group $41-$ 50 . Of the $44 \%$ male respondents, the majority (33.68\%) were in the age group 31-40.

Most of $(51.16 \%)$ the respondents had attained an ordinary level of education and hence had the necessary literacy levels and were able to communicate and interpret information. A total of 43 health facilities were reached through facility assessment although the target had been 57 .

\subsection{VHT Readiness to Implement Mobile Health}

Majority (95.8\%) of the respondents owned a mobile phone with $5.6 \%$ owning more than one. Phone utilization was assessed using the time spent by the VHTs on their mobile phones within the previous 24 hours. Finding indicated that majority $(31.3 \%)$ of the respondents spent less than 30 minutes on their mobile phones. $53.6 \%$ of the respondents spend approximately an hour or less on their mobile phone while over $46 \%$ overall spend more than one hour.

Phone features were grouped into text messaging (71.3\%), internet browsing (18.9\%), gaming (41.4), camera (48.4\%) and other utilities $(65.3 \%)$ for the purpose of measuring knowledgeability on phone usage. Among the other utilities of consideration were the services for mobile payments of bills, purchases of airtime and various utilities as offered by the various mobile MNOs including mobile money applications. Majority $(60.6 \%)$ of the respondents indicated that their favourite feature on the mobile phone is phone texting/messaging.

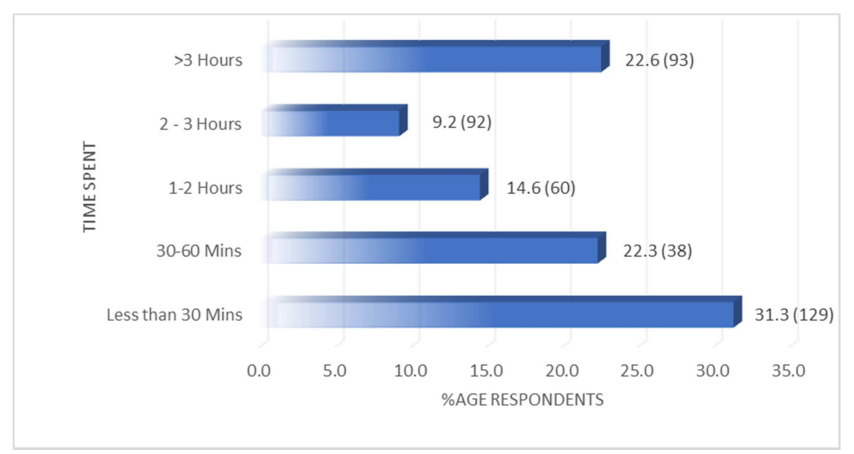

Figure 3. Average time spent on the mobile phone within 24 hours.

\subsection{Telecommunication Assessment}

The telecommunication assessment was based on a consolidation of findings per parish. Cognizant of the fact that majority of the VHTs subscribe to multiple MNOs. The MTN network was reported as the most accessible network in Hoima district with an accessibility of $73 \%$ as reported by the respondents. MTN was still reported as the strongest network for either voice calls or data services with accessibility findings of $88.8 \%$ and $86.2 \%$ respectively.

MNO assessment on data services was necessary because the mHealth applications thrive on the availability of reliable data services provided by MNOs. The network operator of preference for data and internet services was assessed among the VHTs since the mHealth application are mainly based on the sufficient coverage of the data strength of the network operator. The sampled VHTs still maintained MTN as the network operator of choice of preference in their domicile village. Although it reduced by about to value points, it was followed by Airtel which picked up the percentage points in preference as compared to the preferences for voice calls.

The methods by which mobile phones are charged in remote areas was a key consideration as a proxy indicator for maintenance of mobile phones in other areas where the mHealth application has been piloted. The findings on how VHTs in Hoima charge their phones indicated that majority of the VHTs $(92.4 \%)$ have access to solar power with $52.9 \%$ of these accessing it in their households and 39.5\% of these charging their phones at solar powered shops in Hoima town. Only $7.1 \%$ charge using hydroelectricity power commonly called Umeme.

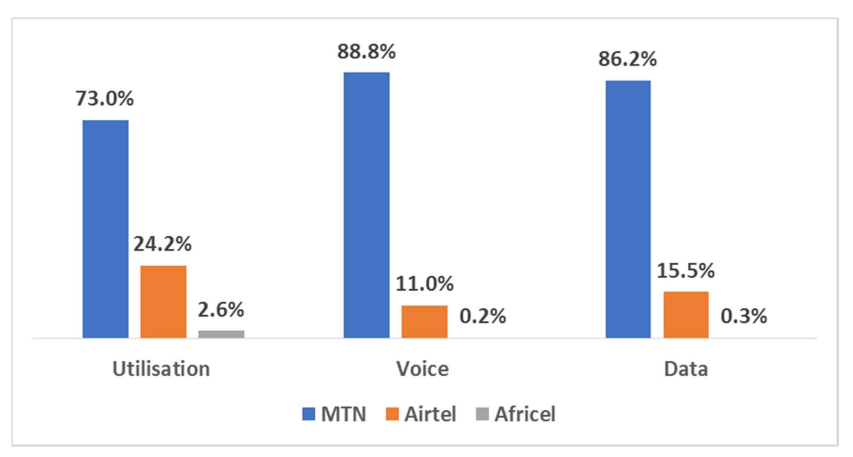

Figure 4. MNOs preferred by the respondents in Hoima.

\subsection{Health Facility Readiness to Implement e-Health}

Analysis of the enabling environment for the mHealth solution at HF level was leveraged on the computer skills of the HF personnel, availability of a functional DHIS2 system and a provision for lighting systems by hydro and/or solar installed powering systems. Majority (55.8\%) of the HFs had personnel trained in computer skills. Only $46.5 \%$ of the trained personnel were proficient in basic computer skills. Also, important to note, the majority of the HFs lacked a powered internet enabled computer and modem; with only $20.9 \%$ reporting availability of a powered internet enabled computer and modem at the HFs.

The $\mathrm{MOH}$ had set up a structure at the district which consisted of the DHIS2 at district level and the HMIS at HF level. The personnel at HF level had been trained to use the DHIS2 and were empowered with access to the HMIS. Only $44.2 \%$ of the staff at the HFs had been trained on the DHIS2, 
and only $37.2 \%$ of the staff had access to the HMIS and are able to send reports using this system.

Majority $(81.4 \%)$ of the sampled HFs required further power installations as they neither had access to a power connection on the national hydroelectric power grid nor solar connection. Only $41.9 \%$ of the HFs had a connection to the national grid power; $48.8 \%$ had a solar installation although these could not meet all the facility needs in form of lighting and appliance management needs.

The HF personnel had their computer skills assessed because of the requirement for good operational knowledge of computers for the mHealth intervention at HF level. The computer skills assessment was categorized into basic computer operation, internet-based applications operation and Microsoft word processing skills. Generally, over $50 \%$ of the HF personnel could operate a computer very well; this was inclusive of internet-based applications operation and Microsoft word processing skills.

$54 \%$ of sampled health workers in the assessed facilities, could ably use a keyboard, a mouse and turn on/off a computer and monitor. Opening a program from the desktop icon and minimizing or maximizing the program windows proficiency was registered among $50 \%$ of the VHTs. Those that fared well across all the categories of general computer skills assessment ranged between $16.7 \%$ at the highest to $8.3 \%$ relatively corresponding to the areas with the lowest and highest scores respectively.

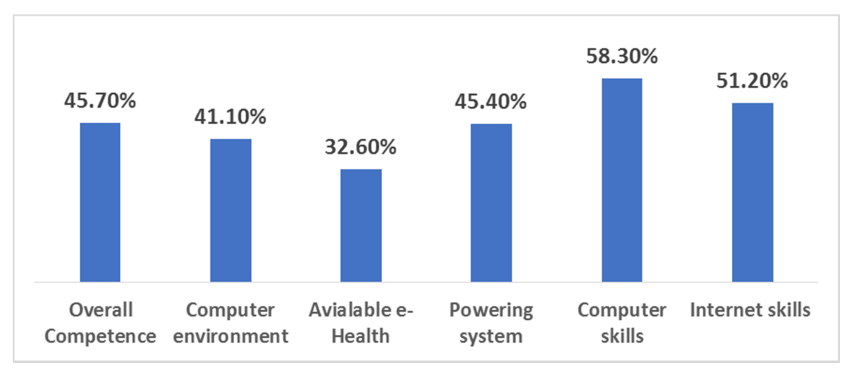

Figure 5. Health facility readiness to implement e-Health.

\subsection{Functional Analysis on the Key e-health Axis and Domains}

The mHealth assessment for scale-up was based on a standard model with a questionnaire that consisted of 16 domains further grouped into 6 axes on which consolidated scores were derived. Axis one that focused on ground work planning for implementation of e-health interventions scored overall $63.7 \%$, contributed by three domains including domain 1 that focused on parameters of Scale measures that scored $100 \%$, domain 2 that focused on Contextual environment that scored $54.5 \%$ and domain 3 focused on Scientific Basis for the e-health implementation scored $36.7 \%$.

For axis two, efforts were made to explore the mHealth project collaborations with the external firms, partners or groups to support the venture of scaling up. It also went further to explore strategies for identifying, developing and sustaining fruitful partnerships and engagements and the overall score for the mHealth project under partnerships was $33.7 \%$ with two domains including domain 4 focusing on Strategic collaborations for the scaling-up process scoring $46.7 \%$ and domain 5 focusing on partnership Sustainability with a score of $20 \%$.

For Axis three relating to financial health, the overall potential costs for estimating the economic costs or scaling up the mHealth project are yet to be forecast. This will be in terms of total costs of ownership, for hardware, software, equipment, training, marketing staffing, identify key cost drivers, identify areas of achieving economies of scale or other cost saving means and outline the assumptions and corresponding risks in forecasting the economic costs over the next three years or so. The overall score for the financial health axis was 30\% with two key domains including domain 6 focusing on financial Management finding scoring $48.9 \%$ and domain 7 focusing on financial modelling results with $11.1 \%$.

For axis four focusing on Technology \& Architecture explored the various steps taken by the project team to optimize the mHealth product for scaling based on its anticipated user base, purpose, integration with information system and compatibility with other components of the information systems architecture. With an overall score for the mHealth project under this axis being $45.7 \%$ with three domains including domain 8 focused on data assessment results with $22.2 \%$, domain 9 focused on interoperability results with $66.7 \%$, and domain 10 focusing on adaptability with $48.1 \%$.

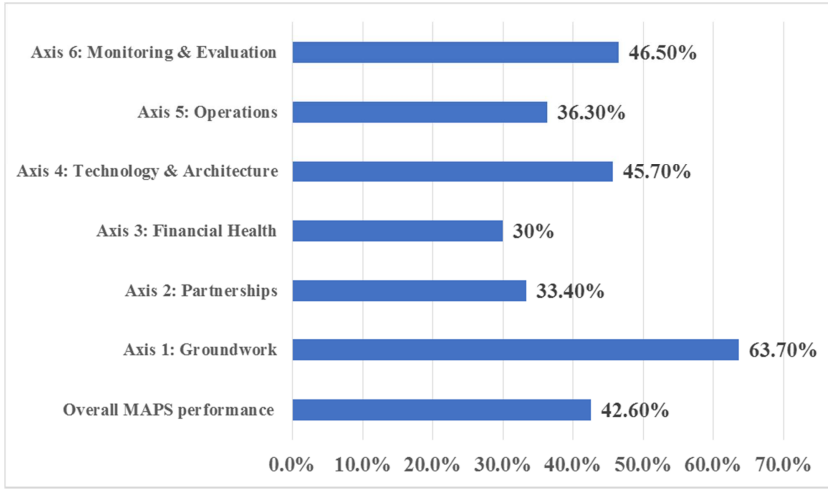

Figure 6. Shows performance of Hoima by the 6 axes of functionality.

Axis 5 focusing on Operations was hinged on for domains that address the personnel issues of development and leadership, training and support, outreach and sensitization and contingency planning. On the overall, the axis scored $36.3 \%$ as contributed to by the following three individual domains including domain 12 focusing on Training and Support with $57.8 \%$, domain 13 focusing on outreach and sensitization with $40 \%$ and domain 1 focusing on contingency Planning with $11.1 \%$.

For axis 6 focusing on monitoring and evaluation, efforts were focused on assessing the decisions and activities that enable effective process monitoring and in-depth outcome evaluation, based on project and stakeholders needs to be undertaken with the overall score for the mHealth project 
under this axis being $46.5 \%$. This had 2 domains including domain 15 focused on process Monitoring assessment results with $40 \%$, and domain 16 focused on evaluation Research assessment results with $53.0 \%$.

\section{Discussion}

The World Health Organization (WHO) has defined mHealth as the use of mobile and wireless technologies to support the achievement of health objectives. The word "support" in this definition is key, because mHealth is most appropriately understood as a tool for promoting healthy behaviours and strengthening health systems. E-health on the other hand is the transfer of health resources and health care by electronic means [19]. It encompasses three main areas: The delivery of health information, for health professionals and health consumers, through the Internet and telecommunications [20].

MHealth at World Vision Uganda is embedded in a global business model that highlights the design and deployment of an open source enterprise wide customized MOTECH Suite solution in partnership with key donors, mHealth providers at national level $(\mathrm{MoH})$ and individual health application developers. The CommCare is uploaded in the mobile phones and takes a community system strengthening approach to improve Maternal, Newborn and Child Health. The mHealth intervention outfits the VHTs with mobile phones enabled with the CommCare application to address the $\mathrm{MNCH}$ issues among the most in need and vulnerable beneficiaries in these areas.

Owing to the positive outcomes elsewhere, WVU seeks to scale-up and employ the same intervention model in Hoima district. It however, sought to perform an mHealth baseline survey and assessment for scale-up of the Hoima RMNCH project before the deployment of the mHealth intervention model. This baseline and scale-up assessment was a prerequisite for the execution of the district wide $\mathrm{RMNCH}$ project aimed at providing an information base against which to assess the applicability of mHealth interventions to improve $\mathrm{MNCH}$ in Hoima district. It is also believed that this baseline survey would help stakeholders party to the Hoima MNCH to understand the value of the mHealth interventions.

The assessment for mHealth scale-up was standardized and consisted of questions relevant to the $16 \mathrm{KPAs}$ for the assessment of scale-up of an mHealth platform. These KPAs are further categorized into six axes which reflect the key concerns and activities that relate to each KPA. The axes are Groundwork, Partnerships, Financial health, Technology \& architecture, Operations and Monitoring\& Evaluation.

The sampled respondents were tasked to make recommendations on how to have a viable mHealth intervention. Many gave recommendations that were tied to strengthening the health system by suggesting provision of computers, human resource staff, construction of health facilities, provision of solar and hydroelectricity options, provision of drugs and other logistics. Other recommendations were directly enabling them to carry out their work and support any mHealth application that would be deployed. With this they requested for training, being equipped with transport facilitation, allowances, kits that include boots uniforms, bags, umbrellas, raincoats, phones and solar chargers to enable them carry out their work.

From the study, 9\% of the respondents indicated that they did not know if their collaborative partnerships favour scalability with government partners, where qualitative signal strength was conducted before rolling out the mHealth application, whether data collection forms underwent formal process optimization analysis and negotiation to achieve streamlining and consolidation, if there is a training manual in place for the mHealth application and if there is cost sharing between the facilities and community on mHealth interventions. Otherwise save for cost sharing between facilities and community, and the organization deploying the mHealth intervention, the rest of the prerequisite indicators are registering over $60 \%$ of response for those facilities currently running with the mHealth intervention.

Majority of the respondents indicated that conducting in-depth mHealth application orientation for all stakeholders was very important for a functional mHealth intervention. Provision of computers to the health facilities and mobile phones for the health workers and VHTs was also sighted as very vital in rolling out a functional mHealth intervention. Computers and phones should be provided to help in moving health services and other work effectively but would also mean installing more electricity and solar panels.

The goal for scaling up was sought by tasking the participants to describe the goals for scaling up in terms of number of health workers, clinics and or clients/beneficiaries reached, number of districts etcetera. The core health outcomes that the mHealth intervention seeks to address is an $\mathrm{MNCH}$ continuum of care with emphasis on the first 1000 days of life. The following responses guarantee the definiteness of goal and purpose ensured.

To empower and strengthen Community Health Systems for positive $\mathrm{MNCH}$ outcomes by increased target number of beneficiaries reached, more districts covered. Reach to 36 additional health facilities and 589 additional VHTs and one new district. Increase on coverage of VHTs and Health Facilities and the population reached after scaling up (1200 VHTs, $57 \mathrm{HFs}$ and over 240,000 beneficiaries). Scale up to all sub counties (15), 35 HFs; 26,170 new born; 105,726 women of child bearing age; 1,222 VHTs.

The team has done work on identifying the health system constraints i.e. challenges the application will seek to address. The project intends to address the information, quality, availability, utilization and demystify some of the negative cultural norms associated with health data. The proposed endgame strategy is the adoption and integration with the $\mathrm{MoH}$ Community Health Management System (CHMS). Growth in VHT/CHW users Increase in women and children under 2 reached/tracked Increase in health facilities supported and linked. It will also consider the Head account as well as number of districts and provinces against total number listed for the country. 
Risks and challenges: For purposes of sustainability in relation to phone replacement and charging, a balance needs to be sought whereby the mobile phones which will be provided to the VHTs are not "too smart". This implies that they should have the minimum requirements to handle the mHealth application but with a good processing speed. Otherwise the mobile phones will continuously run out of battery resulting into a lag for data collection as only $52.9 \%$ of them have a power source at home. The danger in having very "smart" phones also results in the misuse of the phones whereby VHTs can install applications that are attractive for use in their free time and hence deviate from the core purpose of the mobile phones.

Despite tests having been done to transfer the product to different types of mobile devices and operating systems; it is yet to be identified if the product can have its content adapted to new user group. E.g. translation of the content into different languages; and modification of the content based on literacy levels of users and new cultural contexts. Translation of content into different local languages is specifically important because VHTs who may not be conversant with the English language can be able to operate the applications using a language of which they have a better command.

HFs need to have personnel who are proficient in computer skills, a powered computer and an internet connection so as to be able to access the interface within which they can view the data collected by the VHTs in real time. As reported in the findings, over $80 \%$ of HFs do not have access to a reliable and adequate power source. There is a need for solar installations at the HFs. Computers need to be provided to the HFs and so does a modem and recurrent data packages for interrupted internet services.

Recommendations: There is need to adopt a three-tier implementation plan that should bring into consideration the design and preparation stage, the refinement and iteration stage and the training and pilot stage with their related tasks. Technology recommendations have been made for a wide range of android smartphones, solar installations and interoperability approaches. To further harness strategic partners like the MNOs and the $\mathrm{MoH}$ and its related partners, the project should further support operationalization of the DHIS2 by facilitating its linkage to national HMIS at the $\mathrm{MoH}$ headquarters and the CHMS.

For purposes of sharing the values of the mHealth interventions, it is preferred that there be a staff at WVU office who is directly responsible for the data collected by this application; and that this data be shared with partners so as to show the relevance of the mHealth intervention. It is ideal that the HFs have a dedicated staff responsible for the information relayed on the mHealth interface. Generally, most HFs have one or two health workers, and these may not be sufficient for handling patients and managing the interface in parallel if information is to be relayed in real-time by the VHTs. There is a need to have a streamlined and effective mHealth product support arm both at Hoima district regional office and at the national office. At least one staff at the Hoima district regional office should be a single reference point for technical issues of the mobile device and application; and user support.

\section{Conclusion}

The mHealth application has been tested on various mobile devices and operating systems and is able to work on a variety of platforms. The core health outcomes that the mHealth intervention seeks to address is an $\mathrm{MNCH}$ continuum of health care with emphasis on the first 1000 days can surely ride on the suggested mHealth applications if the gaps with identified by the MAPS through the different Axis and domains become the focus for improvement.

Table 3. Domain scores.

\begin{tabular}{ll}
\hline Axis 1: Groundwork & $63.70 \%$ \\
Domain 1: Parameter of Scale & $100 \%$ \\
Domain 2: Contextual environment & $54.50 \%$ \\
Domain3: Scientific basis & $36.70 \%$ \\
Axis 2: Partnerships & $33.40 \%$ \\
Domain 4: Strategic engagement & $46.70 \%$ \\
Domain 5: Partnership sustainability & $20 \%$ \\
Axis 3: Financial Health & $30 \%$ \\
Domain 6: Financial management & $48.90 \%$ \\
Domain 7: Financial model & $11.10 \%$ \\
Axis 4: Technology \& Architecture & $45.70 \%$ \\
Domain 8: Data & $22.00 \%$ \\
Domain 9: Interoperability & $66.70 \%$ \\
Domain 10: Adaptability & $48.10 \%$ \\
Axis 5: Operations & $36.30 \%$ \\
Domain 11: Personnel & $36.40 \%$ \\
Domain 12: Training and Support & $57.80 \%$ \\
Domain 13: Outreach and sensitization & $40 \%$ \\
Domain 14: Contingency planning & $11.10 \%$ \\
Axis 6: Monitoring \& Evaluation & $46.50 \%$ \\
Domain 15: Process Monitoring & $40 \%$ \\
Domain 16: Evaluation Research & $53.00 \%$ \\
\hline
\end{tabular}

\section{Data Availability}

Data is available as an attachment to this article.

\section{Contributions}

(I) Conception and design: G Babughirana, B Manyasi; (II) Administrative support: B Musasizi; (III) Provision of study material or patients: JF Nsumba, RM Mirembe, L Adeke; (IV) Collection and assembly of data: E Nangosha, RM Mirembe, JF Nsumba, L Adeke; (V) Data analysis and interpretation: All authors; (VI) Manuscript writing: All authors; (VII) Final approval of manuscript: All authors.

\section{Competing Interests}

The authors declare that they have no competing interests.

\section{Acknowledgements}

The funding for this research was supported by The World Vision International, Uganda Office, Hoima Cluster from the Hoima Maternal Newborn and Child project. All Health 
facilities, and VHTs who participated are acknowledged.

\section{References}

[1] Polly Walker, et al. 2018, Timed and targeted counselling for health and Nutrition.

[2] WVI, 2016, Facilitator's Guide to 7-11 Health Information: Working in maternal and child health, nutrition, HIV and AIDS, and water, sanitation and hygiene.

[3] Dimagi, 2020, Comcare: The world's most powerful mobile data collection platform. https://www.dimagi.com/commcare/

[4] CHMIS, 2020, Mobile Technology for Community Health (MoTeCH).

[5] WHO, 2011, mHealth New horizons for health through mobile technologies.

[6] Charlene Reynolds, 2013, According to mHealth and MNCH: State of the Evidence. MCHIP-Maternal and Child Health Integrated Program 1776 Massachusetts Avenue. https://www.mchip.net/interventions/mhealth/.

[7] MEASURE Evaluation, 2017, Scaling mHealth for Community-Based Health Information Systems - Lessons and Best Practices.

[8] Leading age, 2020, Strategic Planning and Strategic IT Planning for Long-Term and Post-Acute Care (LTPAC) Providers: A "How To" Workbook.
[9] The MAPS Toolkit: mHealth Assessment and Planning for Scale. Geneva: World Health Organization; 2015.

[10] UDHS, 2016, Uganda demographic and health Survey report 2016.

[11] Rornald M. Kananura, et al, 2016, The neonatal mortality and its determinants in rural communities of Eastern Uganda. https:/www.ncbi.nlm.nih.gov/pmc/articles/PMC4756421/.

[12] Mary Kisakye, 2016, mHealth ttC for AIM Health end term situation analysis report in Busia and Kabale districts.

[13] Hoima District, 2020, From Wikipedia, the free encyclopedia.

[14] Ministry of Finance and planning, 2019, Hoima district approved work plan 2019/2020.

[15] HEALTH SECTOR DEVELOPMENT PLAN2015/16 2019/20.

[16] Acup, C., et al., Factors in fluencing passive surveillance for T. b. rhodesiense human African trypanoso-miasis in Uganda.

[17] The MAPS Toolkit, 2015, mHealth Assessment and Planning for Scale. Geneva: World Health Organization.

[18] KISH, L. 1965. Survey Sampling, John Wiley and Sons, Inc.

[19] FROM INNOVATIONTO IMPLEMENTATION. eHealth in the WHO European Region. World Health Organization 2016.

[20] Oh H, Rizo C, Enkin M, Jadad A. What is eHealth (3): a systematic review of published definitions. J Med Internet Res. 2005; 7 (1): e1. Published 2005 Feb 24. doi: 10.2196/jmir.7.1.e1. 